\title{
The New Dual Antiplatelet Therapy Agents and their Role in Acute Coronary Syndrome
}

\author{
Gareth John Williams ${ }^{1}$ \\ ${ }^{1}$ Medical Student, The Peninsula College of Medicine and Dentistry \\ Correspondence email: garethw512@doctors.net.uk
}

\begin{abstract}
Background: Prasugrel and ticagrelor are two novel antiplatelet agents, which have been subject to large randomized trials to compare their efficacy with clopidogrel for patients with acute coronary syndrome (ACS).

Aim: To conduct a systematic review of prasugrel and ticagrelor as alternative therapy to clopidogrel in patients who present with ACS undergoing PCI.

Methods: The articles cited in this paper were searched on PubMed, MEDLINE, and Plymouth University's Metalib database. The search terms used included "dual antiplatelet therapy", "prasugrel", "ticagrelor", and "clopidogrel resistance".
\end{abstract}

Discussion: The main indications for the use of prasugrel based on current understanding are patients presenting with acute STEMI referred for primary PCI, ACS patients with DM, or those who have a high risk of stent thrombosis. Ticagrelor, on the other hand, may provide optimal benefit for patients with NSTEMI treated with conservative or invasive therapy, those with previous TIA or stroke, advanced age, or small body surface.

Conclusion: Prasugrel and ticagrelor have been shown to be adequate $\mathrm{P} 2 \mathrm{Y}_{12}$ antiplatelet therapy alternatives to clopidogrel in the management of patients with ACS. While prasugrel and ticagrelor have both been shown to clinically improve platelet inhibition and significantly reduce the incidence of stent thrombosis compared with clopidogrel therapy, both increase the risk of a significant bleeding incident. Both ticagrelor and prasugrel have been shown to be appropriate and effective treatment alternatives for ACS patients who display clopidogrel treatment resistance or failure.

\footnotetext{
Copyright Royal Medical Society. All rights reserved. The copyright is retained by the author and the Royal Medical Society, except where explicitly otherwise stated. Scans have been produced by the Digital Imaging Unit at Edinburgh University Library. Res Medica is supported by the University of Edinburgh's Journal Hosting Service: http://journals.ed.ac.uk

ISSN: 2051-7580 (Online) ISBN: 0482-3206 (Print)

Res Medica is published by the Royal Medical Society, 5/5 Bristo Square, Edinburgh, EH8 9AL

Res Medica, 2014, 22(1): 51-63.

doi:10.2218/resmedica.v22i1.811
}

Williams, G. The New Dual Antiplatelet Therapy Agents and their Role in Acute Coronary Syndrome. Res Medica 2014, 22(1), pp.51-63. doi:10.2218/resmedica.v22i1.811 
The New Dual Antiplatelet Therapy Agents and their Role in Acute Coronary Syndrome

\section{Introduction}

The long-term outcome of stent placement by percutaneous coronary interventions (PCI) for the management of acute coronary syndrome (ACS) is improved significantly by 2 key interventions: the use of high-pressure balloon inflations and the administration of periprocedural dual-antiplatelet therapy (DAPT). Although the benefits of DAPT as a medical treatment of ACS have been proven, its indication is obligatory in this setting to prevent post-stent placement complications, such as stent thrombosis and other ischaemic changes. ${ }^{1}$ The basis of this strategy is the suppression of thromboxane $\mathrm{A}_{2}$ production (by the administration of aspirin) and the addition of a $\mathrm{P} 2 \mathrm{Y}_{12}$-receptor inhibitor, an agent that blocks adenosine diphosphate (ADP)-mediated platelet activation. For many years, the $\mathrm{P}_{2} \mathrm{Y}_{12^{-}}$ receptor inhibitor of choice has been the thienopyridine clopidogrel. Clopidogrel combined with aspirin for 12 months has widely become the standard practice following successful management of ACS with PCI. ${ }^{2,3}$

There are, however, 2 concerns when using clopidogrel. The first is its delayed onset of action, which is due to the 2 -stage activation process involving cytochrome P450 isoenzymes. ${ }^{4}$ The second relates to the increasing evidence of a subset of patients that are clopidogrel hyporesponders or nonresponders, who are found to have delayed and/or insufficient inhibition of platelet function. The mechanism for this variable "clopidogrel resistance", also known as "high on-treatment platelet reactivity (HTPR)", is thought to be due to a number of genetic and non-genetic factors that affect the bioactivation of clopidogrel., ${ }^{5,6}$ An optimal inhibition of platelet function therefore cannot be guaranteed in these patients, especially in those who are carriers of the CYP2C19*2 loss-of-function polymorphism. ${ }^{7}$ This phenomenon has commonly been associated with disastrous and life-threatening sequelae, including stent thrombosis, recurrent myocardial infarction (MI), and cardiovascular death. ${ }^{4}$ Although relatively uncommon, this phenotype encompasses a group of patients whereby clopidogrel is not able to provide adequate platelet suppression and for whom alternative treatment options are required. The goal of this article is to synthesize the most recent and relevant literature for the use of the antiplatelet agents prasugrel and ticagrelor in order to provide up-to-date clinical guidance of their use as alternatives for clopidogrel in patients who present with ACS undergoing PCI. 


\section{Methods}

The articles cited in this paper were reviewed for their relevance to this topic. Search engines used include PubMed, MEDLINE, and Plymouth University's Metalib database. The search terms include "dual antiplatelet therapy", "prasugrel”, "ticagrelor", and "clopidogrel resistance". Primary research articles and reviews, including the PLATO and TRITON TIMI38 , were included for their relevance to this subject. Publications included in the search were in English only. Literature published prior to 2008 was included only if it provided critically relevant information, and the majority of papers were identified in the period from 2008 to present.

\section{Discussion}

\section{Prasugrel}

In recent years, new $\mathrm{P} 2 \mathrm{Y}_{12}$-receptor inhibitors have become licensed for use in the management of ACS. The first of these is prasugrel, a third-generation thienopyridine that irreversibly binds to the P2Y 12 receptor. ${ }^{8}$ Prasugrel is similar to clopidogrel in that it is a prodrug that requires bioactivation to become an active metabolite. However, in contrast to clopidogrel, which requires 2 metabolic steps to become its active metabolite, prasugrel requires only 1 metabolic step, which results in a faster and more consistent antiplatelet effect. Furthermore, prasugrel has been shown to function independently of the loss-of-function genetic variants that are believed to cause the wide variability in effectiveness of platelet inhibition seen in clopidogrel therapy.

The clinical effectiveness of prasugrel as an alternative antiplatelet to clopidogrel in patients with ACS has been extensively studied. In the TRITON TIMI-38 trial, ${ }^{9}$ over 13000 patients with ACS were randomized into 2 treatment groups. One group received a $300 \mathrm{mg}$ loading dose (LD) of clopidogrel followed by a maintenance dose (MD) of $75 \mathrm{mg}$ daily for the whole treatment period of up to 15 months, while the second group received a LD of $60 \mathrm{mg}$ of prasugrel followed by $10 \mathrm{mg}$ MD over the same treatment period. With the exception of patients diagnosed with ST-segmentelevation myocardial infarction (STEMI) in whom study medication was given without knowledge of coronary anatomy, a diagnostic angiogram was obligatory for patients before randomization. The results of this trial found that there was a significant reduction in the primary composite efficacy endpoint (cardiovascular mortality, non-fatal MI, and non-fatal stroke) by $18 \%$ in patients taking prasugrel compared with clopidogrel $(9.9 \%$ vs $12.1 \% ; p<0.001)$. In addition, the rate of definite stent thrombosis was halved in the prasugrel group $(0.88 \%$ vs $2.03 \%$; $p<$ 0.001). These results show that the greater 
antiplatelet potency of prasugrel is able to achieve a more favourable and consistent level of platelet function suppression and thus lead to significant improvements in clinical outcomes.

The drawback that was anticipated with the increased efficacy of prasugrel was its increased bleeding risk. Patients taking prasugrel in this trial were found to have an increased incidence of spontaneous noncoronary artery bypass graft (CABG)related major bleeding hazards compared with the clopidogrel group (TIMI bleeding classification: $2.4 \%$ vs $1.8 \%$; $p=0.03$ ), along with more frequent fatal bleedings $(0.4 \%$ vs $0.1 \% ; p=0.002)$. In particular, patients who underwent urgent $\mathrm{CABG}$ surgery were at a significantly higher absolute risk of perioperative bleeding after the intake of at least 1 dose of prasugrel $(13.4 \%$ vs $3.2 \%$ respectively, $p<0.001)$. Other risk factors, such as low bodyweight $(<60 \mathrm{~kg})$, old age, and previous cerebrovascular events, were also associated with unfavourable outcomes. ${ }^{10}$ In order to assess these drawbacks, a prespecified net clinical benefit analysis was performed (a composite of the primary end points and non-CABG-related TIMI major haemorrhage), which demonstrated a net clinical benefit associated with prasugrel therapy despite the excess bleeding $(12.2 \%$ vs $13.9 \%, p=0.004)$. However, these data clearly demonstrate that the benefits of improved platelet suppression with prasugrel therapy must be weighed against its increased bleeding risk, especially in patients who are at a greater risk of severe bleeding.

Interestingly, it was found that for diabetic patients enrolled in the trial, prasugrel displayed a $28 \%$ relative risk reduction (RRR) of the primary composite endpoint to a statistically significant extent $(12.2 \%$ vs $17.0 \%, p<0.001)$ compared with nondiabetics $(9.2 \%$ vs $10.6 \%, p=0.02$, RRR $13.0 \%) .{ }^{10}$ Diabetics who were treated with prasugrel in this trial were found to have a reduced incidence of MI during the follow up period of $40 \%(p<0.001)$. While the rate of severe TIMI bleedings was increased in prasugrel- vs clopidogrel-treated nondiabetics $(2.4 \%$ vs $1.6 \%, p=0.02)$, it was comparable between prasugrel- and clopidogrel-treated diabetics $(2.6 \%$ vs $2.5 \%$, $p=0.81)$. Therefore, it appears that prasugrel may be able to provide further benefit to diabetic patients who present with ACS, which could result in more favourable clinical outcomes.

\section{Ticagrelor}

Whilst clopidogrel and prasugrel are both thienopyridines and prodrugs that require metabolic activation, ticagrelor, an orally administered cyclopentyl-triazolo-pyrimidine, is a directly active compound which binds reversibly to the $\mathrm{P} 2 \mathrm{Y}_{12}$ receptor. ${ }^{11}$ Similar to prasugrel, ticagrelor has a faster onset of 
action than clopidogrel and provides stronger and more consistent platelet inhibition. Due to its reversibility of action and short half-life (7-8.5 hours, which is similar to the active metabolite of prasugrel: 7.4 hours), a LD of $180 \mathrm{mg}$ and MD of $90 \mathrm{mg}$ twice daily is needed to achieve constant platelet inhibition over time. In contrast to clopidogrel, ticagrelor has been shown to have no variability in efficacy due to genetic factors. ${ }^{12}$

The benefit of ticagrelor therapy compared with clopidogrel in preventing cardiovascular events in ACS patients has been evaluated through the PLATO (platelet inhibition and patient outcomes) trial, a multicentre, double-blind, randomized phase-3 trial including over 18000 patients with ACS (non-ST-segment elevation and ST-segment elevation). ${ }^{13}$ In this trial, patients were randomized regardless of which treatment approach was chosen (interventional, primarily conservative, or conservative only) to either the doses detailed above or clopidogrel starting with a LD of 300-600 mg followed by a $75 \mathrm{mg}$ daily $\mathrm{MD}$ over the full study duration. The results of this trial demonstrated that ticagrelor therapy reduced the rate of the primary endpoint (death from vascular causes, non-fatal MI, or non-fatal stroke) at 12 months (9.8\% vs $11.7 \%, p<0.001)$. Patients treated with ticagrelor also experienced a reduction in definite or probable stent thrombosis $(2.2 \%$ vs $3.0 \%, p=0.014)$. This evidence therefore demonstrates very clear clinical benefit in the use of ticagrelor over clopidogrel in ACS patients.

By using the study-specific protocol of severe bleeds including perioperative CABG-related bleedings, no statistically significant differences were demonstrated between the 2 treatment groups $(11.6 \%$ for ticagrelor vs $11.2 \%$ for clopidogrel, $p<$ 0.43). However, the rate of non-CABG major bleeding was increased significantly with ticagrelor when the PLATO (4.5\% vs $3.8 \%, p=0.03)$ and TIMI criteria $(2.8 \%$ vs $2.2 \%, p=0.025 \%$ ) were applied to these results. In addition, although fatal intracranial bleeding was significantly more frequent in the ticagrelor treatment arm ( $0.1 \%$ vs $0.01 \%)$, overall PLATO-defined fatal bleeding was not significantly different between the 2 treatment groups $(0.3 \%$ vs $0.3 \%, p=0.66)$. Of note, the benefit of ticagrelor was shown to be consistent across different subgroup analyses, such as patients with an initial conservative approach with non-invasive therapy, patients undergoing a planned invasive strategy, and also patients undergoing $\mathrm{CABG}$. ${ }^{14-15}$

A number of non-haematological safety endpoints have been observed in ticagrelor, including higher rates of dyspnoea and ventricular pauses, and increased levels of 
creatinine and uric acid during treatment compared with clopidogrel. The exact mechanism for ticagrelor-related dyspnoea remains unproven, with no cardiac or pulmonary pathology observed. ${ }^{16} \mathrm{~A}$ number of observations from preliminary data have led to the hypothesis that adenosine may play a role in this presentation. Ticagrelor has been observed to inhibit adenosine uptake into erythrocytes by increasing circulating levels of adenosine with a theoretical effect similar to that of intravenous adenosine administration. This results in changes to regional blood flow as observed with dipyridamole, which is also an inhibitor of adenosine uptake. ${ }^{17-18}$ In a study by Burki et al., ${ }^{19}$ the intravenous infusion of adenosine into healthy volunteers induced dyspnoea without any associated bronchoconstriction. This thereby led to the likelihood that the adenosine-induced dyspnoea is due to the stimulation of lung receptors, such as vagal C-fibres. The hypothesis may also account for the increased incidence of ventricular pauses, as adenosine administration can induce sinus bradycardia by producing an atrioventriuclar node block. ${ }^{20}$ Although these side effects have been associated with higher discontinuation rates, they have not shown any significant clinical impact. Therefore, although ticagrelor has been shown to increase the bleeding risk of patients when compared with clopidogrel, its use has not increased the rate of overall severe non-haematological clinical events.

The use of ticagrelor over clopidogrel therapy has been studied in a number of prespecified patient subgroups. Of significant importance is ticagrelor's demonstrated reduction in primary combined endpoint in patients undergoing CABG within 7 days after the last study drug intake. The total mortality, cardiovascular death, and noncardiovascular death were all significantly reduced in ticagrelor patients $(p<0.01, p<$ $0.01, p=0.07$ respectively), while the bleeding risk was similar between the treatment regimens. ${ }^{15}$ Additionally, ticagrelor was especially effective in patients with chronic kidney disease (CKD; creatinine clearance $<60 \mathrm{~mL} /$ minute), where it achieved a RRR of the primary combined endpoint of $21 \%$ over clopidogrel $(17.3 \%$ vs $22 \%$ ), which was more pronounced than in patients with normal renal function (RRR $11 \%, 7.9 \%$ vs $8.9 \%) .{ }^{21}$ Therefore, in light of the reported benefits within these subgroup analyses, the use of ticagrelor should especially be considered in certain ACS patients, such as those undergoing $\mathrm{CABG}$ or those with a history of CKD.

\section{Current guidelines and further considerations}

Due to the significant results of both the TRITON TIMI-38 and PLATO trials, both prasugrel and ticagrelor now appear in the 
current European guidelines for non-STelevation ACS and myocardial revascularization. ${ }^{2,22}$ The new guidance for patients with either STEMI or non-ST eleveation myocardial infarction (NSTEMI) referred for primary PCI (PPCI) now recommends both of the new agents as preferred choices over clopidogrel (IB class recommendation for prasugrel and ticagrelor vs IC for clopidogrel). While ticagrelor is recommended for all patients with NSTEMI regardless of the initial treatment strategy, prasugrel is only recommended for patients once the coronary anatomy has been imaged and for those who are undergoing PCI as per the TRITON TIMI-38 trial design. According to the new European guidelines, clopidogrel should now only be used in ACS patients in cases where there are clear contraindications against the newer agents or if neither are available, and may be considered in elderly patients or those with a high bleeding risk. In patients with stable coronary artery disease and planned PCI, clopidogrel remains the agent of choice. In patients due to undergo non-emergency major surgery (including CABG), prasugrel should be discontinued 7 days before the procedure, and ticagrelor and clopidogrel should be discontinued 5 days before.

Due to the positive outcomes reported from the TRITON TIMI-38 trial and the change in international guidance on the management of patients with ACS and subsequent support from the National Institute of Health and Care Excellence (NICE), ${ }^{23}$ prasugrel has been taken up as the default $\mathrm{P}_{2} \mathrm{Y}_{12}$ inhibitor in over half of the units providing PPCI services in the UK. However, there have been doubts over this shift in clinical practice for a number of reasons. Firstly, there were criticisms of the primary efficacy endpoint used in the TRITON TIMI-38 being poorly defined. ${ }^{24}$ Prasugrel has been shown to significantly reduce the incidence of non-fatal MI when compared with clopidogrel $(7.3 \%$ vs $9.5 \%, p$ $<$ 0.001). However, when taking into account the rates of cardiovascular death between the 2 treatment groups, it was found to be $2.4 \%$ and $2.1 \%$ respectively $(p=$ $0.31 \%$ ). Also, the rate of both fatal and nonfatal stroke between the 2 groups were found to be $1.0 \%$ and $1.0 \%(p=0.93)$. While it cannot be disputed that there is a significant reduction in the incidence of stent thrombosis in patients receiving prasugrel compared with clopidogrel $(1.1 \%$ vs $2.4 \%$ respectively, $p<0.0001$ ), it may be argued that the reduction in non-fatal MI cannot justify the excess rates of fatal MI and severe bleeding incurred by this change.

Similarly, there is a perceived drive to prescribe ticagrelor for either PPCI or for all ACS patients in the wake of data procured from the PLATO trial, which is supported by recent NICE guidance. ${ }^{25}$ However, there are concerns raised over the data for 
ticagrelor therapy produced by this trial. One that has already been discussed is the increased bleeding rate found in patients who did not undergo CABG when compared with the clopidogrel treatment regime, when applying either PLATO study bleeding definitions or TIMI major bleeding criteria $(p=0.03)$. Another refers to the PLATO PPCI subgroup analysis, ${ }^{26}$ which found that the rate of primary endpoint was not found to be significantly different between the ticagrelor treatment group and the clopidogrel group, although there was a trend in favour of the former. There was also no significant difference found in mortality rates between the 2 treatment arms. In light of this analysis, it appears that the evidence that has been presented for the benefit of ticagrelor over clopidogrel may not be as robust as initially thought. Therefore, further clinical data may be required to assuage the concerns that have emerged over the replacement of clopidogrel with either prasugrel or ticagrelor.

A particular issue that has raised significant concerns as a result of the PLATO trial is the effect of geographical variation on the effectiveness of ticagrelor. It was noted that in North America, clopidogrel was associated with a better outcome trend than ticagrelor, whereas the reverse was true in the rest of the world (ROW), with a hazard ratio (HR) of 1.25 in North America compared with the HR of 0.84 overall. $^{27}$ Although the United States and the ROW displayed similar data quality and trial conduct, it was discovered that the median maintenance dose of aspirin, which was decided at the discretion of each centre, varied significantly between the 2 subgroups. It was observed that on discharge, patients in North America were more likely to be receiving high-dose maintenance aspirin, whereas patients who were discharged from the ROW were more likely to be receiving low-dose maintenance aspirin. When analysed, this discrepancy may have accounted for between $80 \%$ to $100 \%$ of the observed regional interaction. A similar geographical trend in high maintenance dose of aspirin was observed in the TRITONTIMI 38 trial, with $66 \%$ of patients in North America receiving high-dose aspirin compared with only $28 \%$ in the ROW, with an odds ratio of 5.19 (95\% CI: 4.72-5.70; $p$ $<0.001) .{ }^{28}$ Despite this significant difference between the 2 subgroups, there was no modification of the clinical effect of prasugrel versus clopidogrel based on discharge aspirin dose with respect to the primary efficacy endpoint

(HR $\mathrm{CVD} / \mathrm{MI} /$ stroke $=0.78[95 \% \mathrm{CI}: 0.64 \%-$ $0.95 \%]$ for aspirin < $150 \mathrm{mg} ; \mathrm{HR}$ $\mathrm{CVD} / \mathrm{MI} /$ stroke $=0.87$ [95\% CI: 0.691.10] for aspirin $>150 \mathrm{mg} ; p=0.48)$.

There is currently no definitive biological explanation for this occurrence, although 
there are several potential hypotheses to explain why aspirin doses may modulate the efficacy of ticagrelor. Aspirin exerts an antithrombotic effect by the inhibition of platelet cyclooxygenase, which in turn reduces thromboxane $A_{2}$ release and additionally inhibits endothelial release of prostacyclin in a dose-dependent fashion at daily doses exceeding $80 \mathrm{mg} .{ }^{29}$ Prostacyclin reduces platelet reactivity and may synergistically contribute to the antiplatelet effects of $\mathrm{P}_{2} \mathrm{Y}_{12}$ inhibitors in vivo, which results in the therapeutic effects of a higher mean level of $\mathrm{P}_{2} \mathrm{Y}_{12}$ inhibition. ${ }^{30}$ The therapeutic effects of a higher mean level of $\mathrm{P} \mathrm{Y}_{12}$ inhibition may be attenuated when endogenous prostacyclin production is inhibited. However, due to the absence of a clear pathological process, the possibility that this is the result of chance alone remains a reasonable consideration. However, there is a possible trend observed in high-dose maintenance aspirin with poorer clinical outcomes when given with ticagrelor. Therefore, in conjunction with the results from this analysis and current guideline recommendations, the use of lowdose maintenance aspirin is likely to be associated with the most favourable outcomes with ticagrelor administration.

A final consideration in this article is the comparison between prasugrel and ticagrelor as to which is the most effective antiplatelet agent. Currently, there is no trial that directly compares the 2 agents for the management of ACS patients, which makes much of the potential differences between the two unresolved. Additionally, due to the varying study designs of the TRITON TIMI-38 and PLATO trials, it is not possible to extrapolate the results for a definitive comparison. Despite these limitations, there are a number of simple comparisons between prasugrel and ticagrelor which can be drawn from these 2 studies. Firstly, while the benefit of prasugrel in the TRITON TIMI-38 trial was exclusively attributed to the reduced rate of non-fatal MI of 89 patients, ticagrelor's reduction on mortality in PLATO of 107 deaths clearly represents a major clinical outcome difference between the 2 trials. As previously discussed, both prasugrel and ticagrelor have been shown to significantly reduce the incidence of stent thrombosis compared with clopidogrel. The number needed to treat (NNT) for prasugrel was calculated to be 77 for preventing stent thrombosis, whereas the NNT for ticagrelor was 143. These results may therefore advocate the use of prasugrel over ticagrelor in patients with a higher risk of developing stent thrombosis (e.g. those with diabetes mellitus (DM) and small stent diameter). Another important consideration is that while both medicines lowered the rate of the primary and secondary endpoints in patients with DM compared with clopidogrel, the 
NNT for DM patients taking prasugrel was 21 compared with 48 for ticagrelor. One may therefore favour the use of prasugrel over ticagrelor for patients with DM who present with ACS and are intended for PCI.

With regard to the different ACS subsets, there seems to be a significant benefit for the use of prasugrel rather than ticagrelor in STEMI patients (NNT 42 vs 71 respectively). On the other hand, ticagrelor was shown to reduce the primary endpoint for NSTEMI patients, but not for those with unstable angina, whereas prasugrel had an effect on both. This reduction in mortality may favour prasugrel administration for patients who present with STEMI and ticagrelor for NSTEMI patients.

In terms of side effects, both therapies have been found to similarly increase the risk of non-CABG-related TIMI major bleeding events compared with clopidogrel. However, CABG-related TIMI major bleedings were significantly more prevalent in prasugrel than ticagrelor. In addition, patients who have high risk characteristics where prasugrel is contraindicated, such as a history of transient ischaemic attack (TIA) or stroke, age $>75$ years, body weight of $<$ $60 \mathrm{~kg}$, ticagrelor therapy may be more suitable. As discussed above, the side effects of ticagrelor administration of dyspnoea and ventricular pauses, which are seldom seen in prasugrel administration, may be a cause for concern in terms of drug discontinuation, along with the fact that ticagrelor is administered twice daily as opposed to once daily in the case of prasugrel. Additionally, there are concerns over the increased rate of cancer, especially in women, with prasugrel therapy in TRITON TIMI-38 compared with clopidogrel. In a review of the TRITON TIMI-38 trial by Floyd JS et al., ${ }^{31}$ it was found that 92 patients had new solid tumours in the prasugrel treatment arm $(1.4 \%)$ as opposed to 64 in the clopidogrel arm $(0.9 \%)$ with a HR of new and worse solid cancers of $1.44(p=0.02)$. Currently, the underlying mechanism of this trend is unclear and no association has previously been made between other antiplatelet drugs such as aspirin, clopidogrel, and ticagrelor with an increased risk of new or worsening neoplasms. However, this matter is worth noting when considering the long-term use of prasugrel, and further research must be undertaken.

In short, the main indications for the use of prasugrel based on current understanding are patients presenting with acute STEMI referred for primary PCI, ACS patients with $\mathrm{DM}$, or those who have a high risk of stent thrombosis. Ticagrelor, on the other hand, may provide optimal benefit for patients with NSTEMI treated with conservative or invasive therapy, those with previous TIA or stroke, advanced age, or small body surface. Additionally, by indirectly comparing the 
PLATO and TRITON TIMI-38 trials, one may infer the superiority of ticagrelor to prasugrel for chronic preventative use when taking into account its absolute mortality reduction, reduced haemorrhagic fatalities, and less CABG-related bleeding, as well as a lack of cancer risk. This may therefore suggest that the optimal treatment regimen would be prasugrel prescribed for the first 30 days due to its clear clinical benefit in the early stages of treatment, and then switched to ticagrelor after 30 days due to its potential superiority in achieving favourable longterm outcomes. However, further study is needed in order to ascertain whether this tandem strategy of prasugrel and ticagrelor would be safe and effective in providing an overall clinical benefit in comparison to each medication on its own.

\section{Conclusion}

Prasugrel and ticagrelor have been shown to be adequate $\mathrm{P}_{2} \mathrm{Y}_{12}$ antiplatelet therapy alternatives to clopidogrel in the management of patients with ACS. The results of the TRITON TIMI-38 and PLATO trials have provided a significant insight into the benefits and drawbacks of the use of both agents. While prasugrel and ticagrelor have both been shown to clinically improve platelet inhibition and significantly reduce the incidence of stent thrombosis compared with clopidogrel therapy, both increase the risk of significant bleeding incidents. This is particularly the case in patients with risk factors such as low body weight, advanced age ( $>75$ years old), or those that are due to undergo CABG. There are certain subgroups of ACS patients that have been especially shown to benefit from these new agents. Prasugrel has been shown to improve clinical outcomes in diabetic ACS patients compared with non-diabetic ACS patients, and ticagrelor has demonstrated beneficial outcomes for ACS patients who are due to undergo $C A B G$ or have a history of CKD. There are some doubts over the data from both trials which suggest that prasugrel and ticagrelor are overall superior treatments to clopidogrel, hence further study is required to confirm this. However, there are a number of limitations with clopidogrel use that both of these new agents are not susceptible to, such as a delayed onset of action and a wide variability in efficacy. Both ticagrelor and prasugrel have been shown to be appropriate and effective treatment alternatives for ACS patients who display clopidogrel treatment resistance or failure. 


\section{Learning Points \\ What is already known}

- The use of clopidogrel in conjunction with aspirin for patients with acute coronary syndromes (ACS) has come under scrutiny due to evidence of its varying levels of efficacy, with reports of severe and sometimes fatal outcomes.

- Two novel antiplatelet agents, prasugrel and ticagrelor, have undergone large randomized trials to compare their efficacy to clopidogrel in the TRITON TIMI38 and PLATO trials respectively.

\section{What this study adds}

- In an indirect comparison of the PLATO and TRITON TIMI-38 trials, ticagrelor is shown to have the greater overall clinical benefit with significantly reduced mortality.

- The main indications for prasugrel use is for patients with acute STEMI, ACS patients with DM, and patients at high risk of stent thrombosis.

- The main indications for ticagrelor use is for patients with NSTEMI, patients with a history of CKD, and in those where prasugrel is contraindicated.

- Both agents have demonstrated a more consistent antiplatelet effect than clopidogrel and have shown to be effective alternatives for patients who are nonresponsive to clopidogrel therapy. 


\section{References}

1. Hobson A, Curzen N. Chapter 22: Current status of oral antiplatelet therapies. In: Redwood S, Curzen N, Thomas M, editors. Oxford Textbook of Interventional Cardiology. Oxford, UK: Oxford University Press; 2010. pp. 379-94.

2. Hamm CW, Bassand JP, Agewall S, Bax J, Boersma E, Bueno H, et al. ESC Guidelines for the management of acute coronary syndromes in patients presenting without persistent ST-segment elevation: the task force for the management of acute coronary syndromes (ACS) in patients presenting without persistent ST-segment elevation of the European Society of Cardiology (ESC). Eur Heart J. 2011 Dec;32(23):2999-3054. DOI: 10.1016/j.jpeds.2013.07.019.

3. Jneid H, Anderson JL, Wright RS, Adams CD, Bridges CR, Casey DE Jr, et al. ACCF/AHA focused update of the guideline for the management of patients with unstable angina/non-ST-elevation myocardial infarction (updating the 2007 guideline and replacing the 2011 focused update). Circulation. 2012 Aug 14;126(7):875-910. DOI: 10.1161/CIR.0b013e318256f1e0.

4. Serebruany VL, Steinhubl SR, Berger PB, Malinin AI, Bhatt DL, Topol EJ. Variability in platelet responsiveness to clopidogrel among 544 individuals. J Am Coll Cardiol. 2005 Jan 18;45(2):246-51. DOI: 10.1016/j.jacc.2004.09.067.

5. Simon T, Verstuyft C, Mary-Krause M, Quteineh L, Drouet E, Méneveau N, et al. Genetic determinants of response to clopidogrel and cardiovascular events. N Engl J Med. 2009 Jan 22;360(4):363-75. DOI: 10.1056/NEJMoa0808227.

6. Tentzeris I, Siller-Matula J, Farhan S, Jarai R, Wojta J, Huber K. Platelet function variability and non-genetic causes. Thromb Haemost. 2011 May;105 Suppl 1:S60-6. DOI: 10.1160/THS11-01-0025.

7. Mega JL, Hochholzer W, Frelinger AL 3rd, Kluk MJ, Angiolillo DJ, Kereiakes DJ, et al. Dosing clopidogrel based on CYP2C19 genotype and the effect on platelet reactivity in patients with stable cardiovascular disease. JAMA. 2011 Nov 23;306(20):2221-28. DOI: 10.1001/jama.2011.1703.

8. Angiolillo DJ, Capranzano P. Pharmacology of emerging novel platelet inhibitors. Am Heart J. 2008 Aug;156(2 Suppl):S10-5. DOI: 10.1016/j.ahj.2008.06.004.

9. Wiviott SD, Braunwald E, McCabe CH, Montalescot G, Ruzyllo W, Gottlieb S, et al. Prasugrel versus clopidogrel in patients with acute coronary syndromes. N Engl J Med. 2007 Nov 15;357(20):2001-15. DOI: 10.1056/NEJMoa0706482.

10. Wiviott SD, Braunwald E, Angiolillo DJ, Meisel S, Dalby AJ, Verggheut FW, et al. Greater clinical benefit of more intensive oral antiplatelet therapy with prasugrel in patients with diabetes mellitus in the trial to assess improvement in therapeutic outcomes by optimizing platelet inhibition with prasugrel - Thrombolysis in Myocardial Infarction 38. Circulation. 2008 Oct 14;118(16):1626-36. DOI: 10.1161/CIRCULATIONAHA.108.791061.

11. Angiolillo DJ, Guzman LA. Clinical overview of promising nonthienopyridine antiplatelet agents. Am Heart J. 2008 Aug;156(2 Suppl):S23-8. DOI: 10.1016/j.ahj.2008.06.006.

12. Wallentin L, James S, Storey RF, Armstrong M, Barratt BJ, Horrow J, et al. Effect of CYP2C19 and ABCB1 single nucleotide polymorphisms on outcomes of treatment with ticagrelor versus clopidogrel for acute coronary syndromes: a genetic substudy of the PLATO trial. Lancet. 2010 Oct 16;376(9749):1320-8. DOI: 10.1016/S01406736(10)61274-3.

13. Wallentin L, Becker RC, Budaj A, Cannon CP, Emanuelsson H, Held C, et al. Ticagrelor versus clopidogrel in patients with acute coronary syndromes. N Engl J Med. 2009 Sep 10;361(11):1045-57. DOI: 10.1056/NEJMoa0904327.

14. James SK, Roe MT, Cannon CP, Cornel JH, Horrow J, Husted S, et al. Ticagrelor versus clopidogrel in patients with acute coronary syndromes intended for non-invasive management: Substudy from prospective randomised PLATelet inhibition and patient Outcomes (PLATO) trial. BMJ. 2011 Jun 17;342:d3527. DOI: 10.1136/bmj.d3527.

15. Held C, Asenblad N, Bassand JP, Becker RC, Cannon CP, Claeys MJ, et al. Ticagrelor versus clopidogrel in patients with acute coronary syndromes undergoing coronary artery bypass surgery: results from the PLATO (Platelet Inhibition and Patient Outcomes) trial. J Am Coll Cardiol. 2011 Feb 8;57(6):672-84. DOI: 10.1016/j.jacc.2010.10.029.

16. Storey RF, Bliden KP, Patil SB, Karunakaran A, Ecob R, Butler K, et al. Incidence of dyspnea and assessment of cardiac and pulmonary function in patients with stable coronary artery disease receiving ticagrelor, clopidogrel, 
or placebo, in the ONSET/OFFSET study. J Am Coll Cardiol. 2010 Jul 13;56(3):185-93. DOI: 10.1016/j.jacc.2010.01.062.

17. Gresele P, Arnout J, Deckmyn H, Vermylen J. Mechanism of the antiplatelet action of dipyridamole in whole blood: modulation of adenosine concentration and activity. Thromb Haemost.1986 Feb 28;55(1):12-8.

18. Björkman JA, Kirk I, van Giezen JJ. AZD6140 inhibits adenosine uptake into erythrocytes and enhances coronary blood flow after local ischemia or intracoronary adenosine infusion (abstr). Circulation. 2007;116:II28.

19. Burki NK, Dale WJ, Lee LY. Intravenous adenosine and dyspnea in humans. J Appl Physiol. 2005 Jan;98(1):18085.

20. DiMarco JP, Sellers TD, Berne RM, West GA, Berladinelli L. Adenosine: electrophysiologic effects and therapeutic use for terminating paroxysmal supraventricular yachycardia. Circulation. 1983 Dec;68(6):1254-63. DOI: 10.1161/01.CIR.68.6.1254.

21. James S, Budaj A, Aylward P, Buck KK, Cannon CP, Cornel JH, et al. Ticagrelor versus clopidogrel in acute coronary syndromes in relation to renal function: results from the Platelet Inhibition and Patient Outcomes (PLATO) trial. Circulation. 2010 Sep 14;122(11):1056-67. DOI: 10.1161/CIRCULATIONAHA.109.933796.

22. Wijns W, Kolh P, Danchin N, Di Mario C, Falk V, Folliguet T, et al. Guidelines on myocardial revascularization: the task force on myocardial revascularization of the European Society of Cardiology (ESC) and the European Association for Cardio-Thoracic Surgery (EACTS). Eur Heart J. 2010 Oct;31(20):2501-55. DOI: 10.1093/eurheartj/ehq277.

23. National Institute for Health and Care Excellence. Prasugrel for the Treatment of Acute Coronary Syndromes with Percutaneous Coronary Intervention (TA182). London, UK: National Institute for Health and Care Excellence; October 2009.

24. Curzen, N. Antiplatelet therapy in acute coronary syndromes: beyond aspirin and clopidogrel. Heart. 2012 Nov;98(22):1617-9. DOI: 10.1136/heartjnl-2012-302299.

25. National Institute for Health and Care Excellence. Ticagrelor for the Treatment of Acute Coronary Syndromes (TA236). London, UK: National Institute for Health and Care Excellence; October 2011.

26. Steg PG, James S, Harrington RA, Ardissino D, Becker RC, Cannon CP, et al. Ticagrelor versus clopidogrel in patients with ST-elevation acute coronary syndromes intended for reperfusion with primary percutaenous coronary intervention. A Platelet Inhibition and Patient Outcomes (PLATO) trial subgroup analysis. Circulation. 2010 Nov 23;122(21):2131-41. DOI: 10.1161/CIRCULATIONAHA.109.927582.

27. Mahaffey KW, Wojdyla DM, Caroll K, Becker RC, Storey RF, Angiolillo DJ, et al. Ticagrelor compared with clopidogrel by geographic region in the platelet inhibition and patient outcomes (PLATO) trial. Circulation. 2011 Aug 2;124(5):544-54. DOI: 10.1161/CIRCULATIONAHA.111.047498.

28. Kohli P, Udell JA, Murphy SA, Cannon CP, Antman EM, Braunwald E, et al. Discharge aspirin dose and clinical outcomes in patients with acute coronary syndromes treated with prasugrel versus clopidogrel: an analysis from the TRITON-TIMI 38 Study (Trial to Assess Improvememnt in Therapeutic Outcomes by Optimizing Platelet Inhibition With Prasugrel-Thrombolysis In Myocardial Infarction 38). J Am Coll Cardiol. 2014 Jan 28;63(3):22532. DOI: $10.1016 /$ j.jacc.2013.09.023.

29. FitzGerald GA, Oates JA, Hawiger J, Maas RL, Roberts LJ 2nd, Lawson JA, et al. Endogenous biosynthesis of prostacyclin and thromboxane and platelet function during chronic administration of aspirin in man. J Clin Invest. 1983 Mar;71(3):676-88. DOI: 10.1172/JCI110814.

30. Cattaneo M, Lecchi A. Inhibition of the platelet P2Y12 receptor for adenosine diphosphate potentiates the antiplatelet effect of prostacyclin. J Thromb Haemost. 2007 Mar;5(3):577-82.

31. Floyd JS, Serebruany VL. Prasugrel as a potential cancer promoter: review of the unpublished data. Arch Intern Med. 2010 Jun 28;170(12):1078-80. DOI: 10.1001/archinternmed.2010.154. 